\title{
Clinical Characteristics Indicating Uterine Leiomyoma, Adenomyosis, and Uterine Leiomyoma Coexisting with Adenomyosis: Retrospective Study
}

\section{Jeong-A Hong, Soo-Ho Chung}

Department of Obstetrics and Gynecology, Soonchunhyang University Bucheon Hospital, Soonchunhyang University College of Medicine, Bucheon, Korea

\begin{abstract}
Objective: This study is to compare the baseline characteristics and symptoms between groups with leiomyoma only (group $M$; myoma group), adenomyosis only (group A; adenomyosis group), and leiomyoma and adenomyosis together (group B; group for both disease).

Methods: Selected patients were who received total abdominal hysterectomy, laparoscopy-assisted vaginal hysterectomy, or total laparoscopic hysterectomy from January 2014 to December 2015, and whose pathology result showed leiomyoma $(n=74)$, adenomyosis $(n=27)$, or both $(n=63)$. Baseline characteristics and symptoms were reviewed from the medical records. Researched characteristics included patients' age, degeneration of leiomyoma, endometrial hyperplasia, endometriosis, weight of the removed uterus, menopause before the surgery, method of the surgery, necessity for blood transfusion before and after the surgery, difference of hemoglobin level before and after the surgery, and number of gravida, para, and abortion.

Results: Eleven symptoms were checked. Thirty-eight point four percent of total subject had uterine leiomyoma and adenomyosis at the same time. Number of abortion was higher in the group B. The group B showed a tendency of presenting more menorrhagia, dysfunctional uterine bleeding, acute lower abdominal pain, and urinary frequency. Symptoms related to mass effect seem to be relative to uterine leiomyoma, and symptoms related to menorrhagia seems to be relative to adenomyosis. The group $M$ showed suddenly growing mass symptoms, and was more likely to have massive hemorrhage during the surgery. It is hard to differentiate coexistence of uterine leiomyoma and adenomyosis from each disease.

Conclusion: Coexistence of two disease exhibits mixed symptoms of each disease, but shows different tendency.
\end{abstract}

Keywords: Adenomyosis; Leiomyoma; Hysterectomy

\section{INTRODUCTION}

Uterine leiomyomas and adenomyosis are the most common gynecologic disorders in reproductive aged women. Uterine leiomyomas are benign monoclonal tumors that originate primarily from smooth muscle cell proliferation in the myometrium. Adenomyosis is a benign condition characterized by invasion of the myometrium by the endometrium and associated with uterine enlargement, and histologically exhibits ectopic, nonneoplastic, endometrial glands and stroma surrounded by the hypertrophic myometrium [1]. Both uterine leiomyomas and adenomyosis are serious gynecological pathologies that may require hysterectomy in premenopausal women. Uterine leiomyomas are most often as- ymptomatic, but may cause a multitude of urinary symptoms such as abnormal uterine bleeding, chronic pelvic pain, urinary frequency, and others [2]. The most common symptoms of adenomyosis are menorrhagia in about $50 \%$ of affected patients and dysmenorrhea in $30 \%$ of patients [3]. Since these two disorders frequently occur concurrently, they cannot be cleanly distinguished by symptoms alone $[4,5]$. Although the symptoms of uterine leiomyomas and adenomyosis have been identified to a great extent, comparative studies on symptoms of these disorders when present separately and concurrently have been rarely done. Therefore, this study intended to compare clinical features and symptoms by classifying patients who underwent hysterectomy into leiomyoma only group, adenomyosis only group, and concomitant leiomyoma 
and adenomyosis group based on their histopathologic outcomes.

\section{MATERIALS AND METHODS}

This research was a retrospective study conducted in patients who underwent hysterectomy in the Department of Obstetrics and Gynecology in Soonchunhyang University Bucheon Hospital, Gyeonggi-do from January 2014 to December 2015, and was approved by the Institutional Review Board of Soonchunhyang University Bucheon Hospital (IRB approval no., SCHBC_2014-01-016).

Of all patients who received total abdominal hysterectomy, laparoscopy-assisted vaginal hysterectomy, and total laparoscopic hysterectomy over the past 2 years, 179 patients with leiomyoma, adenomyosis, or concurrent leiomyoma and adenomyosis were recruited to the study based on their histopathologic findings. Those who underwent hysterectomy due to pregnancy, cervical, ovarian, endometrial, or colorectal cancer, uterine arteriovenous malformation or uterine arteriovenous fistula, and uterine or vaginal prolapse were excluded. Excluding 15 patients (three with cervical cancer, seven with endometrial cancer, and five with ovarian cancer), a total of 164 patients were finally included in this study. They were divided into three groups of myoma uteri (74 cases), adenomyosis (27 cases), and both diseases (63 cases).

Through medical record review, we examined patients' age, the presence of leiomyoma with degeneration, endometrial hyperplasia and endometriosis after surgery, weight of removed uterus, preoperative menopausal status, surgery type, pre- and postoperative transfusion rates, hemoglobin and hematocrit levels measured within 30 days prior to surgery, hemoglobin and hematocrit levels measured on the first postoperative day, changes in hemoglobin and hematocrit levels, the total number of pregnancies and deliveries, the number of incomplete pregnancies including miscarriages and induced abortions, and the number of cesarean sections. Based on medical records, the presence of 11 symptoms (menorrhagia, dysmenorrhea, prolonged menstruation, bleeding other than a normal menstrual period, postmenopausal bleeding, chronic back pain, chronic pelvic pain, rapid growth of a mass, acute lower abdominal pain, a palpable mass in the abdomen, and urinary frequency) was examined. The authors included patients who visited our hospital with a chief complaint of a palpable mass growing rapidly over a short period of time, and those with a chief complaint of recent enlargement of adenomyosis or leiomyomas that had been followed up for a long period with a regular pelvic ultrasound.

Chi-square test or Fisher's exact test was used to compare statistical differences in clinical characteristics of patients who received hysterectomy according to the presence of myoma uteri or adenomyosis alone, or both diseases. Regarding continuous variables, the Shapiro-Wilk test was performed to test for normality and Levene's test was done to test homogeneity of variance.

Based on the results, one-way analysis of variance (ANOVA) or Kruskal-Wallis test was performed to compare the three groups. The Bonferroni was used as a post-hoc test. Based on a two-tailed test, P-values of less than 0.05 were considered statistically significant. All statistical analyses were performed using SPSS Statistics for Windows ver. 14.0 (SPSS Inc., Chicago, IL, USA) and R Statistical Software ver. 3.1.3 (R Foundation for Statistical Computing, Vienna, Austria).

\section{RESULTS}

A total of 164 patients consisted of 74 (45.1\%) in the myoma uteri group, 27 (16.5\%) in the adenomyosis group, and 63 (38.4\%) in the group with both diseases. The mean age was $47.9 \pm 6.8$ years in the myoma uteri group, $48.5 \pm 9.7$ years in the adenomyosis group, and $47.4 \pm 5.1$ years in the group with both diseases. The mean age of all subjects was $47.8 \pm 6.8$ years, displaying no statistically significant difference (Table 1).

No significant differences were found with regard to the presence of leiomyoma with degeneration after biopsy, endometrial hyperplasia, and endometriosis in three groups. The average weight of removed uterus was $570.8 \pm 667.8 \mathrm{~g}$ in myoma uteri group, $335.7 \pm 219.2 \mathrm{~g}$ in the adenomyosis group, and $431.8 \pm 247.3$ $\mathrm{g}$ in the group with both diseases. Although uterine weight tended to be heavier in the myoma uteri group and the group with both diseases having leiomyomas, there was no statistical significance. A giant uterus weighed up to 7 times more than the average weight in the myoma uteri group, but uterus weight did not exceed 1,000 g in the adenomyosis group (Fig. 1).

The three groups had insignificant difference in the frequency of preoperative menopause, and no significant difference in surgery type attempted according to disease.

Pre- or postoperative transfusion rate was $5.4 \%$ in the myoma uteri group, $7.4 \%$ in the adenomyosis group, and $4.8 \%$ in the group with both diseases, showing no statistical difference. The change in hemoglobin level, which was defined as the preoperative hemo- 
Hong JA, et al. • Clinical Characteristics of Uterine Leiomyoma and Adenomyosis

Table 1. Baseline characteristics in patients with myoma, adenomyosis, and both of them

\begin{tabular}{|c|c|c|c|c|c|}
\hline Variable & Myoma $(n=74)$ & Adenomyosis $(n=27)$ & Both $(n=63)$ & Total $(n=164)$ & Comparison (P-value) \\
\hline Age (yr) & $47.9 \pm 6.8$ & $48.5 \pm 9.7$ & $47.4 \pm 5.1$ & $47.8 \pm 6.8$ & 0.926 \\
\hline Myoma degeneration & $1(1.4)$ & 0 & 0 & $1(0.6)$ & 0.542 \\
\hline Endometrial hyperplasia & $1(1.4)$ & $3(11.1)$ & $4(6.3)$ & $8(4.9)$ & 0.103 \\
\hline Endometriosis & $2(2.7)$ & $1(3.7)$ & $3(4.8)$ & $6(3.7)$ & 0.815 \\
\hline Uterine weight (g) & $570.8 \pm 667.8$ & $335.7 \pm 219.2$ & $431.8 \pm 247.3$ & $478.7 \pm 488.6$ & 0.126 \\
\hline Preoperative menstruation & & & & & 0.888 \\
\hline Ongoing & $63(85.1)$ & $24(88.9)$ & 54 (85.7) & $141(86.0)$ & \\
\hline Menopause & $11(14.9)$ & $3(11.1)$ & $9(14.3)$ & $23(14.0)$ & \\
\hline Operative method & & & & & 0.882 \\
\hline TLH & $47(63.5)$ & $19(70.4)$ & $42(66.7)$ & $108(65.9)$ & \\
\hline LAVH & $12(16.2)$ & $5(18.5)$ & $10(15.9)$ & $27(16.5)$ & \\
\hline TAH & $15(20.3)$ & $3(11.1)$ & $11(17.5)$ & $29(17.7)$ & \\
\hline Red blood cell transfusion & $4(5.4)$ & $2(7.4)$ & $3(4.8)$ & $9(5.5)$ & 0.880 \\
\hline \multicolumn{6}{|l|}{ Laboratory factor } \\
\hline Hemoglobin (mg/dL) at baseline & $12.0 \pm 1.9$ & $11.8 \pm 1.9$ & $11.9 \pm 1.8$ & $12.0 \pm 1.8$ & 0.630 \\
\hline Hemoglobin (mg/dL) after operation & $10.1 \pm 1.8$ & $10.7 \pm 1.4$ & $10.1 \pm 1.4$ & $10.2 \pm 1.6$ & 0.143 \\
\hline$\Delta$ Hemoglobin $(\mathrm{mg} / \mathrm{dL})$ & $2.0 \pm 1.7$ & $1.1 \pm 1.2$ & $1.9 \pm 1.4$ & $1.8 \pm 1.5$ & $0.033^{1-2,1-3}$ \\
\hline$\Delta$ Hemoglobin $(\mathrm{mg} / \mathrm{dL})$ in patients without transfusion & $1.9 \pm 1.6$ & $1.3 \pm 1.0$ & $1.9 \pm 1.4$ & $1.8 \pm 1.4$ & 0.200 \\
\hline Hematocrit (mg/dL) at baseline & $37.0 \pm 4.7$ & $35.6 \pm 5.2$ & $36.6 \pm 4.5$ & $36.6 \pm 4.7$ & 0.482 \\
\hline Hematocrit (mg/dL) after operation & $30.7 \pm 5.0$ & $32.1 \pm 3.7$ & $30.7 \pm 3.7$ & $30.9 \pm 4.3$ & 0.235 \\
\hline$\Delta$ Hematocrit (mg/dL) & $6.3 \pm 5.1$ & $3.5 \pm 3.9$ & $6.0 \pm 4.0$ & $5.7 \pm 4.6$ & $0.018^{1-2,1-3}$ \\
\hline$\Delta$ Hematocrit $(\mathrm{mg} / \mathrm{dL})$ in patients without transfusion & $6.3 \pm 4.9$ & $4.2 \pm 3.0$ & $6.1 \pm 4.0$ & $5.9 \pm 4.3$ & 0.146 \\
\hline \multicolumn{6}{|l|}{ Reproductive factor } \\
\hline G (no. of pregnancies) & $2.8 \pm 1.3$ & $3.3 \pm 1.4$ & $3.5 \pm 1.9$ & $3.2 \pm 1.6$ & 0.045 \\
\hline $\mathrm{P}$ (no. of births of viable offspring) & $1.8 \pm 0.8$ & $1.9 \pm 0.8$ & $1.8 \pm 0.7$ & $1.8 \pm 0.7$ & 0.557 \\
\hline L (no. of living children) & $1.8 \pm 0.8$ & $1.9 \pm 0.8$ & $1.8 \pm 0.7$ & $1.8 \pm 0.7$ & 0.557 \\
\hline A (no. of abortion) & $1.0 \pm 1.0$ & $1.4 \pm 1.2$ & $1.7 \pm 1.7$ & $1.4 \pm 1.4$ & $0.018^{2-3}$ \\
\hline No. of cesarean section & $0.4 \pm 0.7$ & $0.7 \pm 0.9$ & $0.6 \pm 0.8$ & $0.5 \pm 0.8$ & 0.375 \\
\hline
\end{tabular}

Values are presented as mean \pm standard deviation for continuous variables and frequency $(\%)$ for categorical variables. P-value was calculated by one-way analysis of variance or Kruskal-Wallis test for continuous variables and chi-square test or Fisher's exact test for categorical variables as appropriate. Post-hoc comparison was conducted using Bonferroni's correction: i-j means that there is statistically significant difference between the i-th group and the j-th group (i, j=1, 2, 3; 1st group, myoma; 2nd group, adenomyosis; 3rd group, both).

TLH, total laparoscopic hysterectomy; LAVH, laparoscopy-assisted vaginal hysterectomy; TAH, total abdominal hysterectomy; G, gravida; P, para; L, living children; A, abortion.

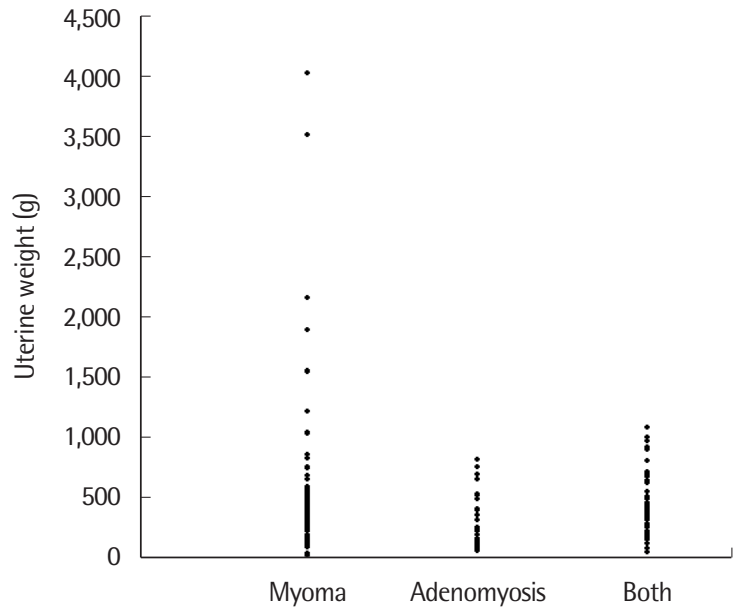

Fig. 1. Distribution of uterine weight in patients with myoma, adenomyosis, and both of them. globin level subtracted from the postoperative hemoglobin level, was $2.0 \pm 1.7 \mathrm{mg} / \mathrm{dL}$ in the myoma uteri group, $1.1 \pm 1.2 \mathrm{mg} / \mathrm{dL}$ in the adenomyosis group, and $1.9 \pm 1.4 \mathrm{mg} / \mathrm{dL}$ in the group with both diseases before excluding the blood transfusion group. The hemoglobin levels were higher in the myoma uteri group and the group with both diseases than the adenomyosis group. Moreover, significant differences were exhibited between the myoma uteri and adenomyosis groups and between the group with both diseases and the adenomyosis group. Though a greater variability was observed in the myoma uteri group and the group with both diseases having leiomyomas compared to the adenomyosis group when patients who received a blood transfusion pre- or postoperatively were excluded, no statistically significant difference across the three groups. 
Table 2. Symptoms in patients with myoma, adenomyosis, and both of them

\begin{tabular}{|c|c|c|c|c|c|}
\hline Variable & Myoma $(n=74)$ & Adenomyosis $(n=27)$ & Both $(n=63)$ & Total $(n=164)$ & Comparison (P-value) \\
\hline Menorrhagia & $38(51.4)$ & $15(55.6)$ & $31(49.2)$ & $84(51.2)$ & 0.858 \\
\hline Dysmenorrhea & $22(29.7)$ & $12(44.4)$ & $29(46.0)$ & $63(38.4)$ & 0.115 \\
\hline Prolonged menstruation & $5(6.8)$ & $3(11.1)$ & $7(11.1)$ & $15(9.1)$ & 0.629 \\
\hline Dysfunctional uterine bleeding & $10(13.5)$ & $5(18.5)$ & $15(23.8)$ & $30(18.3)$ & 0.299 \\
\hline Postmenopausal vaginal bleeding & $2(2.7)$ & $1(3.7)$ & 2 (3.2) & $5(3.0)$ & 0.964 \\
\hline Chronic lower-back pain & $2(2.7)$ & $2(7.4)$ & $3(4.8)$ & $7(4.3)$ & 0.568 \\
\hline Chronic pelvic pain & $6(8.1)$ & $3(11.1)$ & $5(7.9)$ & $14(8.5)$ & 0.871 \\
\hline Suddenly growing mass & $6(8.1)$ & 0 & 0 & $6(3.7)$ & 0.023 \\
\hline Acute lower abdominal pain & $4(5.4)$ & $3(11.1)$ & $9(14.3)$ & $16(9.8)$ & 0.211 \\
\hline Palpable abdominal mass & $9(12.2)$ & 0 & $6(9.5)$ & $15(9.1)$ & 0.170 \\
\hline Urinary frequency & $3(4.1)$ & $1(3.7)$ & $5(7.9)$ & $9(5.5)$ & 0.552 \\
\hline
\end{tabular}

Values are presented as frequency (\%). P-value was calculated by chi-square test or Fisher's exact test as appropriate. Post-hoc comparison was conducted using Bonferroni's correction.

In relation to obstetrical history, the total number of pregnancies (gravida) was $2.8 \pm 1.3$ in the myoma uteri group, $3.3 \pm 1.4$ in the adenomyosis group, and $3.5 \pm 1.9$ in the group with both diseases on average. When post-hoc tests were conducted after a oneway ANOVA to compare the three groups, no significant difference was found.

The average number of abortions (abortus) was $1.0 \pm 1.0$ in the myoma uteri group, $1.4 \pm 1.2$ in the adenomyosis group, and $1.7 \pm$ 1.7 in the group with both diseases. There was a significant difference between groups $(\mathrm{P}=0.018)$. According post-hoc analysis, a significant difference was shown between the myoma uteri group and the group with both diseases. The number of abortions tended to be higher in the adenomyosis group and the group with both diseases having adenomyosis. The average number of cesarean sections was $0.4 \pm 0.7$ in the myoma uteri group, $0.7 \pm 0.9$ in the adenomyosis group, and $0.6 \pm 0.8$ in the group with both diseases. A higher tendency towards cesarean section was detected in the adenomyosis group and the group with both diseases compared to the myoma uteri group. However, no statistical difference was found.

In comparison of 11 symptoms, menorrhagia occurred at similar rates in all three groups (Table 2). The prevalence of dysmenorrhea was $29.7 \%$ in the myoma uteri group, $44.4 \%$ in the adenomyosis group, and $46.0 \%$ in the group with both diseases. Although the groups having adenomyosis had a higher rate of occurrence, there was no statistical significance.

Prolonged menstruation occurred at similar rates in all three groups. The prevalence of dysfunctional uterine bleeding was $13.5 \%$ in the myoma uteri group, $18.5 \%$ in the adenomyosis group, and $23.8 \%$ in the group with both diseases. Though the highest frequency was seen in the group with both diseases, there was no statistical difference. Postmenopausal bleeding, chronic back pain, and chronic pelvic pain were manifested similarly in all groups. A rapidly growing mass occurred in $8.1 \%$ of patients in the myoma uteri group, presenting a statistically significant difference between groups $(\mathrm{P}=0.023)$. A mass growing rapidly over a short period of time was detected in six cases, and all cases belonged to the myoma uteri group. The occurrence rate of acute lower abdominal pain was $5.4 \%$ in the myoma uteri group, $11.1 \%$ in the adenomyosis group, and $14.3 \%$ in the group with both diseases. Patients in the adenomyosis group and the group with both diseases were more likely to complain of acute lower abdominal pain, but had no statistical significance. The incidence of a palpable mass in the abdomen was $12.2 \%$ in the myoma uteri group, $0 \%$ in the adenomyosis group, and $9.5 \%$ in the group with both diseases. Although the groups having leiomyomas had a higher incidence of a palpable mass in the abdomen, no statistical significance was found. Urinary frequency was manifested similarly in all groups.

\section{DISCUSSION}

Uterine leiomyomas are benign monoclonal tumors that originate from smooth muscle cell proliferation in the myometrium. In an American study, when ultrasound scans were performed in a population of randomly selected women, leiomyomas were detected in $60 \%$ of 35 -year-old and $80 \%$ of 50 -year-old African Americans, and in $40 \%$ of 35 -year-old and $70 \%$ of 50 -year-old White Americans [2]. The development of leiomyomas is identified to be related to hormonal and genetic factors and growth fac- 
Hong JA, et al. • Clinical Characteristics of Uterine Leiomyoma and Adenomyosis

tors [6]. Uterine leiomyomas are often a symptomatic, but may cause abnormal uterine bleeding including menorrhagia, chronic pelvic pain, and urinary symptoms such as urinary frequency, urinary urgency or nocturia, and others. On the contrary, a cohort study reported that the size or number of leiomyomas had no significant impact on menstrual cycles or characteristics [7]. The diagnosis of leiomyomas is usually determined by pelvic examination and imaging tests such as pelvic ultrasound or magnetic resonance imaging scans. Asymptomatic leiomyomas are typically observed with serial follow-ups, while symptomatic leiomyomas can be managed by drug therapy using a hormone-releasing intrauterine device, interventions such as uterine artery embolization, and surgical modalities such as hysteromyomectomy or hysterectomy [8].

Adenomyosis is a common benign condition characterized by invasion of ectopic endometrium into the myometrium [5]. The prevalence estimates vary widely from study to study, ranging from $5 \%$ to $70 \%$, and the median rate is approximately $20 \%$ to $30 \%$ $[9,10]$. Hyperestrogenemia related to endometrial hyperplasia or myometrium diseases is also linked to adenomyosis. In addition, previous pregnancy history is related to adenomyosis. The occurrence of endometriosis is also known to be associated with adenomyosis [5]. The most common symptoms of adenomyosis are abnormal uterine bleeding, chronic pelvic pain, and dysmenorrhea [5]. Patients with these symptoms can be suspected of having adenomyosis, and often undergo hysterectomy. Drug, interventional, or surgical management can be attempted for patients with adenomyosis. Even though sex hormones, progestins, danazol, and gonadotropin-releasing hormone can help alleviate dysmenorrhea, several controlled studies have shown that no drug has inhibited the development of adenomyosis [11,12]. An interventional modality is artery embolization similar to leiomyomas, and possible surgical approaches are hysteroscopic endometrial ablation, myomectomy, hysterectomy, and others [13].

In the present study, no significant difference was found with regard to mean age across the three groups. Although we hypothesized that myoma degeneration is related to pain development in patients due to necrosis based on our histological findings, this symptom was present in a patient only in the myoma uteri group.

Excluding patients who received pre- and postoperative transfusions, no statistically significant changes between pre- and postoperative hemoglobin levels were observed. However, a greater significance was found in the groups with leiomyomas. We can infer that a great difference between pre- and postoperative hemoglobin levels implies high intraoperative blood loss. A larger volume of intraoperative blood loss is projected in the groups with leiomyoma alone or concurrent leiomyoma compared to the group with adenomyosis alone.

There was a significant difference in the total number of incomplete pregnancies (the sum of miscarriages and induced abortions). A higher difference was observed in the groups having adenomyosis and concomitant adenomyosis. Even though the pathogenesis of adenomyosis has not yet been sufficiently clarified, adenomyosis develops when the endometrium invades the myometrium.

Some studies have suggested that the risk of endometriosis increases when the border of the myometrium and endometrium is invaded after surgical techniques [14]. In cases of induced or spontaneous abortion, dilatation and curettage may damage the endometrium, basement membrane, and myometrial wall, and this seems to result in an increased frequency of adenomyosis. Although the number of cesarean sections was not statistically significant, a greater risk of adenomyosis was found in the adenomyosis group and the group with both diseases than the myoma uteri group, and this may be also attributable to the same reason.

No significant difference was observed with respect to several symptoms between groups. Although dysmenorrhea showed no significant difference, a tendency to suffer from dysmenorrhea was highest in the group with both diseases, followed by adenomyosis group and myoma uteri group. Patients with adenomyosis were more likely to complain of menstrual cramps than those with leiomyomas. Moreover, patients with both diseases tended to complain of menstrual cramps more frequently. The group with both diseases had a higher tendency to have vaginal bleeding other than a normal menstrual period, acute lower abdominal pain and urinary frequency than the other two groups. The adenomyosis group had the highest frequencies of dysmenorrhea, chronic back pain, chronic pelvic pain, and other pain-related items.

All patients with a rapidly growing mass over a short period of time were identified to have leiomyomas. A mass palpable in the abdomen was detected only in the myoma uteri group and the group with both diseases, indicating that mass-related symptoms are related to uterine leiomyomas.

Leiomyomas and adenomyosis share many of the same symptoms. However, different drug therapies are available including the use of levonorgestrel-releasing intrauterine system and ulipris- 
tal acetate to manage leiomyomas, and dienogest to treat adenomyosis, in addition to pain relief. We initiated this study with the belief that associated symptoms may be useful in determining the underlying disease to a certain degree.

As we began this study, we hypothesized that more distinctive symptoms will manifest in patients with concurrent leiomyoma and adenomyosis compared to those with leiomyoma or adenomyosis alone. The results of this study have revealed that although a differential diagnosis of leiomyoma from adenomyosis can be difficult based on symptoms alone, several clinical features have been detected. The size of leiomyomas was quite large in the leiomyoma group. On the other hand, leiomyoma size did not exceed $1,500 \mathrm{~g}$, the uterus was palpable during physical examination, and the rapid growth of a mass was unseen in the group with both diseases. Miscarriage rate was significantly higher in the group with both diseases, and this is thought to be an important cause for an increased prevalence of adenomyosis in the group with both diseases. Even though no statistical difference was found in regard to patient's symptoms, symptoms in the group with both diseases were similar to those of the adenomyosis group including dysmenorrhea, prolonged menstrual bleeding, and bleeding other than a normal menstrual period. These symptoms appear to be caused by adenomyosis rather than leiomyomas. To sum up the above findings, clinical manifestations of leiomyomas and adenomyosis can overlap and vary by coexistence of these two conditions.

This retrospective study was limited in certain aspects. The presence of symptoms was identified, but the severity of symptoms was not evaluated. Furthermore, the current study retrospectively reviewed medical records, and a pathologist excluded very small coexisting lesions at the time of postoperative histopathological examination. For this reason, there is a possibility of excluding patients with both diseases in the concomitant leiomyoma and adenomyosis group. Finally, since the subjects were not grouped according to uterus size, the difference in symptoms according to uterus size was not examined. Future prospective studies will help overcome these limitations by comparing symptoms according uterus size and the degree of symptom expression and severity of symptoms across different groups classified according to postoperative histopathological outcomes after inquiring patients suspected of leiomyomas or adenomyosis using questionnaires with items on 11 or more symptoms and evaluating the severity of symptoms expressed on a numeric rating scale.

\section{REFERENCES}

1. Garcia L, Isaacson K. Adenomyosis: review of the literature. J Minim Invasive Gynecol 2011;18:428-37.

2. Parker WH. Etiology, symptomatology, and diagnosis of uterine myomas. Fertil Steril 2007;87:725-36.

3. Templeman C, Marshall SF, Ursin G, Horn-Ross PL, Clarke CA, Allen M, et al. Adenomyosis and endometriosis in the California Teachers Study. Fertil Steril 2008;90:415-24.

4. Weiss G, Maseelall P, Schott LL, Brockwell SE, Schocken M, Johnston JM. Adenomyosis a variant, not a disease?: evidence from hysterectomized menopausal women in the Study of Women's Health Across the Nation (SWAN). Fertil Steril 2009;91:201-6.

5. Flake GP, Andersen J, Dixon D. Etiology and pathogenesis of uterine leiomyomas: a review. Environ Health Perspect 2003;111:1037-54.

6. Marino JL, Eskenazi B, Warner M, Samuels S, Vercellini P, Gavoni N, et al. Uterine leiomyoma and menstrual cycle characteristics in a populationbased cohort study. Hum Reprod 2004;19:2350-5.

7. Sabry M, Al-Hendy A. Medical treatment of uterine leiomyoma. Reprod Sci 2012;19:339-53.

8. Yeniel O, Cirpan T, Ulukus M, Ozbal A, Gundem G, Ozsener S, et al. Adenomyosis: prevalence, risk factors, symptoms and clinical findings. Clin Exp Obstet Gynecol 2007;34:163-7.

9. Taran FA, Stewart EA, Brucker S. Adenomyosis: epidemiology, risk factors, clinical phenotype and surgical and interventional alternatives to hysterectomy. Geburtshilfe Frauenheilkd 2013;73:924-31.

10. Wood C. Surgical and medical treatment of adenomyosis. Hum Reprod Update 1998;4:323-36.

11. Fernandez H. New concepts on pathophysiology, diagnosis and treatment of adenomyosis. J Gynecol Obstet Biol Reprod (Paris) 2003;32(8 Pt 2): S23-7.

12. Levgur M. Therapeutic options for adenomyosis: a review. Arch Gynecol Obstet 2007;276:1-15.

13. Levgur M, Abadi MA, Tucker A. Adenomyosis: symptoms, histology, and pregnancy terminations. Obstet Gynecol 2000;95:688-91.

14. Panganamamula UR, Harmanli OH, Isik-Akbay EF, Grotegut CA, Dandolu V, Gaughan JP. Is prior uterine surgery a risk factor for adenomyosis? Obstet Gynecol 2004;104(5 Pt 1):1034-8. 